\title{
A Comparison of The Effects of Clobetasol 0.05\% and Photodynamic Therapy Using Aminolevulinic Acid With Red Light in the Treatment of Severe Nail Psoriasis
}

\author{
Zohreh Tehranchinia ${ }^{1}$, Nasibeh Barzkar ${ }^{1}$, Seyed Mohammad Riahi ${ }^{2}$, Marjan Khazan ${ }^{1 *}$ \\ ${ }^{1}$ Skin Research Center, Shahid Beheshti University of Medical Sciences, Tehran, Iran \\ ${ }^{2}$ Social Determinants of Health Research Center, Department of Epidemiology and Biostatistics, Faculty of Health, Birjand \\ University of Medical Sciences, Birjand, Iran
}

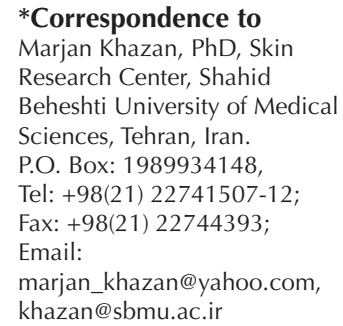

\begin{abstract}
Introduction: The treatment of nail psoriasis is often difficult and unsatisfactory due to therapy resistance. This study aimed to compare the efficacy of clobetasol $0.05 \%$ ointment and photodynamic therapy (PDT) using aminolevulinic acid (ALA) and red light in the treatment of severe nail psoriasis. Methods: This open-trial study included 69 nails of 8 patients with severe nail psoriasis. Thirty-four nails were treated with clobetasol $0.05 \%$ ointment and 35 nails were treated with ALA-PDT. ALAPDT treatment was performed every 3 weeks for 5 cycles; the nails were treated with clobetasol under occlusion every day during the study. All the patients were followed up after 24 weeks. The severity of nail involvement was measured by the Nail Psoriasis Severity Index (NAPSI) scores at the baseline and during the treatment.

Results: There were no significant differences in the NAPSI scores between the treatment groups in the baseline and weeks 3,6 and 9 (all $P>0.05$ ), although significant differences were found in weeks 12,15 and 24 (follow-up) $(P<0.001)$. A significant time-effect improvement was found in all the nailmatrix, nail-bed and total NAPSI scores in both treatment groups (all $P<0.001$ ). The patients did not report intense pain and discomfort during irradiation.

Conclusion: Clobetasol $0.05 \%$ ointment seems to be effective in treating nail psoriasis after a treatment period of 15 weeks. However, the efficacy of ALA-PDT at a 24-week follow-up was greater than that of clobetasol.

Keywords: Nail psoriasis; Photodynamic therapy; Aminolevulinic acid; Red light; Clobetasol.
\end{abstract}

\section{Introduction}

Psoriasis is a common chronic inflammatory skin disorder with nail involvement, which is mostly estimated to be $80 \%$ of psoriatic patients during lifetime ${ }^{1}$; however, psoriasis can just be limited to the nails in $5 \%-10 \%$ of cases. ${ }^{2}$ On the basis of the nail structure, the clinical manifestations are mainly due to the involvement of nail matrices, such as pitting, leukonychia, and ridging. In addition, they can be because of the involvement of nail beds, including oil drop patches, onycholysis, splinter hemorrhages, and subungual hyperkeratosis. ${ }^{3}$ It has been shown that nail changes in patients with nail psoriasis are associated with cosmetic problems, interference with work, and pain, which may consequently lead to a negative impact on the quality of life. ${ }^{2,45}$ Additionally, nail psoriasis can be considered as a predictor of psoriatic arthritis. ${ }^{6}$ Therefore, although nail psoriasis treatment can be challenging and unsatisfactory due to therapy resistance, safe and effective treatments are required to improve the quality of life and reduce the risk of psoriasis-triggering diseases. ${ }^{5}$ It has been shown that due to the limited efficacy of the topical therapies in nail psoriasis because of the low penetrability of the topical therapies in the nail matrix and nail bed, ${ }^{3}$ phototherapy as a systemic therapy may be effective in nail psoriasis. ${ }^{7}$ Recently, photodynamic therapy (PDT) with 5-aminolevulinic acid (ALA) as a photosensitizer has been shown to be effective in treatment for some skin diseases, including sebaceous hyperplasia, acne, and psoriasis..$^{8-11}$ PDT with red light may improve the permeability of the nail bed and nail matrix by using ALA, which is metabolized to protoporphyrin IX and accumulation of ALA-induced protoporphyrin IX can be

Please cite this article as follows: Tehranchinia Z, Barzkar N, Mohammad Riahi S, Khazan M. A comparison of the effects of clobetasol 0.05\% and photodynamic therapy using aminolevulinic acid with red light in the treatment of severe nail psoriasis. J Lasers Med Sci. 2020;11(1):3-7. doi:10.15171/jlms.2020.02. 
a target for PDT. ${ }^{12,13}$

Although several studies have reported the efficacy of PDT in the treatment of plaque-type psoriasis, ${ }^{8,11}$ there are not enough previous studies that show the effectiveness of PDT in nail psoriasis. ${ }^{10}$ Consequently, the present study aimed to assess and compare the clinical efficacy of clobetasol propionate $0.05 \%$ ointment and PDT using ALA and red light in the treatment of nail psoriasis.

\section{Materials and Methods}

Populations of Study

This open-trial study included 69 nails of 8 outpatients aged $\geq 20$ with moderate-to-severe fingernail psoriasis from the Dermatology Department of Shohada-e Tajrish Hospital between November 2016 and May 2017. The diagnosis was according to the clinical symptom and the direct potassium hydroxide smear of nail lesions; direct microscopy and culture were performed to exclude onychomycosis in some problematic patients. The exclusion criteria comprised patients with nail psoriasis, who had received topical and/or systemic therapies within the last 3 months, history of photosensitivity, pregnancy, and lactation.

The severity of nail psoriasis was assessed by a blinded dermatologist during treatment using the Nail Psoriasis Severity Index (NAPSI) score, which was used for the nail bed, the nail matrix, and the total nail of each fingernail. According to the NAPSI score, the nail was divided into quarters, then each quadrant was evaluated for the presence or absence of any of features of the nail bed and the nail matrix. The maximum possible score was 8 for each fingernail (4 for matrix plus 4 for bed scores). ${ }^{14}$ NAPSI scores and photographs were performed and taken at the start of each session (at the beginning and at every 3 weeks for 5 cycles) during the treatment. At least a $25 \%$ reduction in NAPSI scores from the baseline was considered a positive response to treatment. ${ }^{15}$ The improvement of each fingernail was based on the following scores between 0 and 6: $0=100 \%$ improvement (total clearance); $1=90 \%$ improvement (almost total clearance); $2=75 \%$ improvement (distinct clearance); $3=50 \%$ improvement (moderate clearance); $4=25 \%$ improvement (mild clearance); $5=$ no change; and $6=$ worse.

\section{Study Protocol}

The right hand as an experimental group was treated with ALA and red light-PDT, and the left hand as a control group was treated only with clobetasol propionate $0.05 \%$ ointment, which was massaged into the nail bed and covered with an occlusive dressing to increase its efficacy for 15 weeks every day. First, the nail lesions of the right hand were sealed with 20\% ALA gel (MEDAC GmBH Hamburg) for 3 hours to induce the accumulation of protoporphyrin IX, as a photosensitizing agent, in the nail that was confirmed by UV irradiation. Then, PDT with the red-light source (PDT 1200, Waldmann Medizintechnik, Villingen-Schwenningen, Germany) was applied one time at a wavelength of $630 \mathrm{~nm}$ (range $600-730 \mathrm{~nm}$ ) with a total dose of $120 \mathrm{~J} / \mathrm{cm}^{2}$ and an intensity of $200 \mathrm{~mW} / \mathrm{cm}^{2}$ for approximately 20 minutes. This treatment was performed every 3 weeks for 5 sessions. Follow-up evaluation was done 24 weeks after the last treatment session. To reduce and manage pain during irradiation, a mini hand-held fan was used near to the site of treatment.

Statistical Analysis

The Shapiro-Wilk test was used to assess the normal distribution. The Student's $t$ test was used to compare the groups. The differences between the last treatment session and 24-week follow-up in both groups were determined by the paired-samples $t$ test. Repeated measurement analysis of variance was used to compare the mean of the NAPSI score during the treatment. When the Mauchly's test of sphericity showed the heterogeneity of covariance, the Greenhouse-Geisser correction was performed to adjust the degrees of freedom in the F-tests. All statistical analyses were done by the SPSS 22.0 version, and statistically significant results were considered as $P<0.05$.

\section{Results}

From a total of 69 severe psoriatic nails, 35 were treated with ALA-PDT and 34 with clobetasol propionate $0.05 \%$ in a group of 8 patients with a mean age of $32.23 \pm 5.49$.

The mean of the nail matrix, the nail bed, and the total NAPSI scores at the baseline and in weeks 4, 8, 15 (end of treatment), and 24 (follow-up) are shown in Figure 1.

No significant differences were found in the mean of total NAPSI scores between the two groups at the baseline and in weeks 3,6 , and $9(P=0.862, P=0.915$, $P=0.698, P=0.695$ respectively ) (Figurela); there were also no significant differences in nail bed and nail matrix NAPSI scores between the two treatment groups at the baseline ( $P=0.466, P=0.389$ respectively) and in weeks 3 ( $P=0.240, P=0.314$ respectively), $6(P=0.234, P=0.513$ respectively), and $9(P=0.477, P=0.751$ respectively) (Figure $1 \mathrm{~b}$ and 1c). However, statistically significant differences were observed in all three scores in weeks 12 and $15(P<0.001)$ between the treatment groups.

The repeated measurement analysis of variance with a Greenhouse-Geisser correction showed that during the treatment period, a statistically significant reduction was found in the means of nail matrix, nail bed and total NAPSI scores in the treatment groups (all $P<0.001$ ) (Figure 1); a significant time-effect improvement was also found in all three scores in both groups (all $P<0.001$ ). The mean of total NAPSI score between two groups at the baseline and the 15-week was statistically significantly different $(F=8.472, P=0.005)$.

The mean NAPSI scores in nails treated with ALA-PDT significantly decreased from $5.97 \pm 1.29$ at the baseline to $4.29 \pm 1.44$ in week 15 and $2.11 \pm 1.27$ at the end of week 

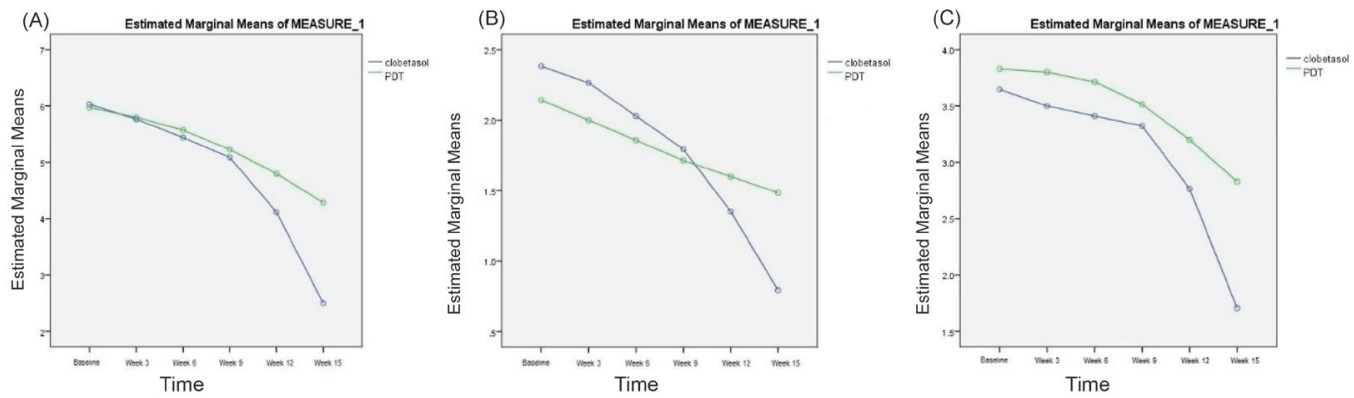

Figure 1. The Comparison of Mean NAPSI Scores in PDT and Clobetasol Groups. (A) the mean total nail NAPSI score in both treatment groups. (B) the mean nail matrix NAPSI score in both treatment groups. (C) the mean nail bed NAPSI score in both treatment groups.

24 of follow-up $(P<0.001)$. Clobetasol in the treatment of nail psoriasis showed a significant reduction in the mean NAPSI scores from the baseline $(6.03 \pm 1.47)$ to week 15 of treatment $(2.50 \pm 1.05)(P<0.001)$ and an increase in the mean NAPSI scores from $2.50 \pm 1.05$ in week 15 to $3.94 \pm$ 0.95 in the follow-up $(P<0.001)$ (Table 1$)$. There were also significant differences in the means of the nail matrix and nail bed scores from the baseline to the end of treatment and in the follow-up in both groups (all $P<0.001$ ).

At the end of the 15-week treatment period, the mean NAPSI was reduced to $2.50 \pm 1.05$ presenting a $58 \%$ improvement in the clobetasol group and $4.29 \pm 1.44$ presenting a $28 \%$ improvement in the ALA-PDT group.

At the 24-week follow-up after the last treatment session, the mean NAPSI score was significantly greater in the nails treated with ALA-PDT than in the nails treated with clobetasol $(P<0.001)$ (Figure 2$)$.

None of the patients reported intense pain and discomfort during irradiation.

\section{Discussion}

The results of this study showed significant differences in the mean NAPSI scores between ALA-PDT and clobetasol $0.05 \%$ ointment at weeks 12,15 and 24 . Clobetasol has been shown to be more effective in the treatment of severe psoriatic nails than ALA-PDT, although the treatment efficacy at a 24-week follow-up was significantly greater in the nails treated with ALA-PDT compared with the nails treated with clobetasol.

The incidence of nail psoriasis is reported among approximately $50 \%$ of patients and consequently can cause a decreased quality of life among them. ${ }^{1,2,45}$ Nail psoriasis could also potentially be related to the risk factor in psoriatic arthritis. ${ }^{6}$ First line therapies are often topical treatments, although their efficacy in nail psoriasis is limited due to the low penetrability of the nail bed and nail matrix. ${ }^{3}$ However, the effectiveness of topical therapies on the nail plate may be increased under occlusion. ${ }^{16}$ The clobetasol propionate at a concentration of $0.05 \%$ is the most common topical agent in the treatment of psoriasis. ${ }^{17}$ The long-term use of clobetasol propionate is associated with adverse effects such as skin atrophy, telangiectasia, depigmentation and bone reabsorption. ${ }^{18}$ Although it has been shown that clobetasol $0.05 \%$ in cream/gel/ ointment generally has poor efficacy in the treatment of nail psoriasis due to limited penetration, ${ }^{16}$ Rigopoulos et al showed the significant efficacy of clobetasol $0.05 \%$ cream in improving nail psoriasis 12 weeks after treatment. ${ }^{19}$ Similarly, in this study, clobetasol propionate $0.05 \%$ ointment was shown to significantly improve nail psoriasis after a 15-week treatment period. However, Nakamura et al found a significantly positive effect of clobetasol propionate $8 \%$ on nail lacquer, when compared the efficacy of clobetasol at different concentrations of $0.05 \%, 1 \%$, and $8 \%$ in patients with nail psoriasis. ${ }^{20}$ These contrasting findings are probably due to different

Table 1. The Nail Psoriasis Severity Index (NAPSI) Scores During the Treatment and the Follow-Up Period

\begin{tabular}{|c|c|c|c|c|c|c|}
\hline \multirow{2}{*}{ Week } & \multicolumn{3}{|c|}{ PDT-Treated Nails $(\mathrm{n}=35)^{*}$} & \multicolumn{3}{|c|}{ Clobetasol-Treated Nails $(n=34)^{*}$} \\
\hline & Total & Bed & Matrix & Total & Bed & Matrix \\
\hline 0 (baseline) & $5.97 \pm 1.29$ & $3.83 \pm 0.85$ & $2.14 \pm 1.19$ & $6.03 \pm 1.46$ & $3.65 \pm 1.17$ & $2.38 \pm 1.10$ \\
\hline 3 & $5.80 \pm 1.25$ & $3.80 \pm 0.86$ & $2.00 \pm 1.11$ & $5.76 \pm 1.47$ & $3.50 \pm 1.21$ & $2.26 \pm 1.05$ \\
\hline 6 & $5.57 \pm 1.33$ & $3.71 \pm 0.92$ & $1.86 \pm 1.03$ & $5.44 \pm 1.44$ & $3.41 \pm 1.15$ & $2.03 \pm 1.14$ \\
\hline 9 & $5.23 \pm 1.39$ & $3.51 \pm 1.01$ & $1.71 \pm 0.98$ & $5.09 \pm 1.56$ & $3.32 \pm 1.19$ & $1.79 \pm 1.09$ \\
\hline 12 & $4.80 \pm 1.38$ & $3.20 \pm 1.10$ & $1.60 \pm 0.91$ & $4.12 \pm 1.32$ & $2.76 \pm 1.25$ & $1.35 \pm 0.95$ \\
\hline 15 & $4.29 \pm 1.44$ & $2.83 \pm 0.95$ & $1.49 \pm 0.91$ & $2.50 \pm 1.05$ & $1.71 \pm 0.97$ & $0.79 \pm 0.64$ \\
\hline 24 (follow-up) & $2.11 \pm 1.27$ & $1.34 \pm 0.87$ & $0.89 \pm 0.75$ & $3.94 \pm 0.95$ & $2.29 \pm 0.87$ & $1.79 \pm 0.91$ \\
\hline
\end{tabular}

Abbreviation: PDT, photodynamic therapy.

All variables represent Mean \pm SD. ${ }^{*} P$ values $<0.05$ were considered significant. 


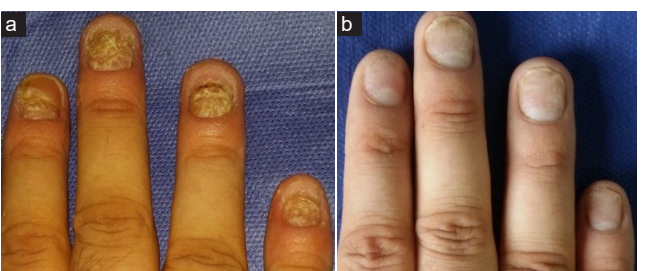

Figure 2. Effect of ALA-PDT Therapy on the Nail in one of the Patients Showing Moderate Clearance. (a) Before PDT treatment. (b) After a 24-week follow-up.

settings, patient diversity, differences in ethnicity, gender, age, psychosocial factors, nutritional status, smoking, and environmental factors as mentioned in other studies. ${ }^{21-24}$

On the other hand, in recent years PDT has been described to be effective in the treatment of plaque-type psoriasis, ${ }^{9,25}$ while there are not enough prior studies reporting the treatment of nail psoriasis with PDT. Fernández-Guarino et al showed the efficacy of PDT and the pulsed dye laser in the treatment of nail psoriasis, although the reduction in the NAPSI score in both treatment groups was not significant; they revealed $21 \%$ improvement in the NAPSI score after 3 months and 54\% improvement after 6 months of treatment with PDT. ${ }^{10}$ In addition, they found that methyl-aminolevulinic acid (MAL) did not play any role in treating nail psoriasis. Similarly, according to this study, nails treated with ALAPDT showed a $28 \%$ improvement in the NAPSI score in week 15, and no patient showed discomfort during the sessions despite slight pain. Thus, PDT treatment was well tolerated by the patients. In contrast, low efficacy and a high percentage of pain have been shown in the treatment of psoriasis with PDT, as has been supported by the British Association of Dermatologists guidelines. ${ }^{26}$

Finally, our findings at the end of the 6-month followup showed that clinical improvement in the NAPSI score was achieved with ALA-PDT treatment, while the cases treated with clobetasol $0.05 \%$ showed worsening or recurrence of symptoms of nail psoriasis.

The factors influencing the efficacy of PDT and pain intensity during PDT include light sources, radiation doses, and ALA concentrations. ${ }^{27}$ Different light sources can be currently employed in PDT, such as laser, red lights, blue lights, intense pulsed light, light-emitting diodes, and other visible lights. ${ }^{17}$ Photosensitizers of PDT include MAL, ALA, methylene blue, etc. Recently, ALA and MAL in different concentrations are applied in PDT, which were widely used to treat oncological conditions such as actinic keratosis and also some inflammatory dermatoses such as psoriasis, sebaceous hyperplasia, hidradenitis suppurativa, and acne vulgaris, ${ }^{8,9}$ although there is no study to assess the efficacy of various light sources of PDT in different concentrations of ALA/MAL for the treatment of nail psoriasis.

Psoriasis is a chronic, relapsing disease, and the management of nail psoriasis is challenging and difficult due to resistance in therapy and/or slow response to the treatment because of slow-growing nails, which can be affected by age and nutrient deficiency. Thus it requires long-standing treatment that might increase the risk of medication side effects. On the other hand, there is no standardized treatment for nail psoriasis and the selected treatment is dependent on multiple factors such as the history of the patient, age, sex, severity, the duration of nail psoriasis, concomitant diseases and medications, and the risks and benefits of therapy. Furthermore, systemic treatment is not recommended in psoriatic patients with only nail manifestations; it is suggested in patients with skin or joint involvement or patients resistant to treatment. $^{16}$

For these reasons, in this study, it seems that ALA and red light-PDT might be an effective and safe therapeutic choice for the treatment of severe nail psoriasis due to long-term effects (at the end of follow-up) without discomfort and severe pain during treatment and any side effects

Our study has some limitations. This study had a small sample size and was not randomized and double-blind. In addition, growing nails are strongly associated with nutrients intake that was not assessed in the patients using the Food Frequency Questionnaire.

\section{Conclusion}

After the last treatment session, the mean NAPSI scores in the nails treated with clobetasol $0.05 \%$ ointment were significantly less than those in the nails treated with ALAPDT; however, 6 months after the last treatment session, the mean NAPSI scores in the nails treated with ALA-PDT were greater than those in the nails treated with clobetasol $0.05 \%$ ointment. Treatments were also well tolerated and none of the patients complained of discomfort during the sessions except slight pain. Additional studies using a larger number of patients are needed to confirm these results.

\section{Ethical Considerations}

The informed consent forms were taken from all patients. The study protocol (identifier: IRCT2015112825271N1; https://www.irct.ir/trial/21161) received approval from the Ethics Committee of Shahid Beheshti University of Medical Sciences (Ethical Code: IR.SBMU.SRC. REC.1394.1).

\section{Conflict of Interests}

The authors declare no conflict of interest.

\section{References}

1. Greb JE, Goldminz AM, Elder JT, Lebwohl MG, Gladman DD, Wu JJ, et al. Psoriasis. Nat Rev Dis Primers. 2016;2:16082. doi: 10.1038/nrdp.2016.82.

2. Dogra A, Arora AK. Nail Psoriasis: The Journey So Far. Indian J Dermatol. 2014;59(4):319-333. doi: 10.4103/0019- 
5154.135470.

3. Jiaravuthisan MM, Sasseville D, Vender RB, Murphy F, Muhn CY. Psoriasis of the nail: anatomy, pathology, clinical presentation, and a review of the literature on therapy. J Am Acad Dermatol. 2007;57(1):1-27. doi: 10.1016/j. jaad.2005.07.073.

4. Ortonne JP, Baran R, Corvest M, Schmitt C, Voisard JJ, Taieb C. Development and validation of nail psoriasis quality of life scale (NPQ10). JEur Acad Dermatol Venereol. 2010;24(1):22-27. doi: 10.1111/j.1468-3083.2009.03344.x.

5. Haneke E. Nail psoriasis: clinical features, pathogenesis, differential diagnoses, and management. Psoriasis (Auckl). 2017;7:51-63. doi: 10.2147/PTT.S126281.

6. Raposo I, Torres T. Nail psoriasis as a predictor of the development of psoriatic arthritis. Actas Dermosifiliogr. 2015;106(6):452-457. doi: 10.1016/j.ad.2015.02.005.

7. Lawry M. Biological therapy and nail psoriasis. Dermatol Ther. 2007;20(1):60-67. doi: 10.1111/j.15298019.2007.00112.x.

8. Megna M, Fabbrocini G, Marasca C, Monfrecola G. Photodynamic Therapy and Skin Appendage Disorders: A Review. Skin Appendage Disord. 2017;2(3-4):166-176. doi: 10.1159/000453273.

9. Zhang L, Wu Y, Zhang Y, Liu X, Wang B, Wang P, et al. Topical 5-aminolevulinic photodynamic therapy with red light vs intense pulsed light for the treatment of acne vulgaris: A spilit face, randomized, prospective study. Dermatoendocrinol. 2017;9(1):e1375634. doi: 10.1080/19381980.2017.1375634.

10. Fernández-Guarino $\mathrm{M}$, Harto $\mathrm{A}$, Sánchez-Ronco M, García-Morales I, Jaén P. Pulsed dye laser vs. photodynamic therapy in the treatment of refractory nail psoriasis: a comparative pilot study. J Eur Acad Dermatol Venereol. 2009;23(8):891-895. doi: 10.1111/j.14683083.2009.03196.x.

11. Choi YM, Adelzadeh L, Wu JJ. Photodynamic therapy for psoriasis. J Dermatolog Treat. 2015;26(3):202-207. doi: 10.3109/09546634.2014.927816.

12. Stringer MR, Collins P, Robinson DJ, Stables GI, SheehanDare RA. The accumulation of protoporphyrin IX in plaque psoriasis after topical application of 5-aminolevulinic acid indicates a potential for superficial photodynamic therapy. J Invest Dermatol. 1996;107(1):76-81. doi: 10.1111/15231747.ep12298282.

13. Krutmann J, Hönigsmann H, Elmets CA, Bergstresser PR, editors. Dermatological Phototherapy and Photodiagnostic Methods. Berlin: Springer; 2009.

14. Rich P, Scher RK. Nail Psoriasis Severity Index: a useful tool for evaluation of nail psoriasis. J Am Acad Dermatol. 2003;49(2):206-212. doi: 10.1067/s0190-9622(03)00910-1.

15. Cannavò SP, Guarneri F, Vaccaro M, Borgia F, Guarneri B. Treatment of psoriatic nails with topical cyclosporin: a prospective, randomized placebocontrolled study. Dermatology. 2003;206(2):153-156. doi: $10.1159 / 000068469$.

16. Piraccini BM, Starace M. Optimal management of nail disease in patients with psoriasis. Psoriasis (Auckl). 2015;5:25-33. doi: 10.2147/PTT.S55338.

17. Rigopoulos D, Ioannides D, Prastitis N, Katsambas A. Nail psoriasis: a combined treatment using calcipotriol cream and clobetasol propionate cream. Acta Derm Venereol. 2002;82(2):140. doi: 10.1080/00015550252948220.

18. Wolf R, Tur E, Brenner S. Corticosteroid-induced 'disappearing digit'. J Am Acad Dermatol. 1990;23(4):7556. doi: 10.1016/s0190-9622(08)81079-1.

19. Rigopoulos D, Gregoriou S, Katsambas A. Treatment of psoriatic nails with tazarotene cream $0.1 \%$ vs. clobetasol propionate $0.05 \%$ cream: a double-blind study. Acta Derm Venereol. 2007;87(2):167-8. doi: 10.2340/00015555-0195.

20. Nakamura RC, Abreu L, Duque-Estrada B, Tamler C, Leverone AP. Comparison of nail lacquer clobetasol efficacy at $0.05 \%, 1 \%$ and $8 \%$ in nail psoriasis treatment: prospective, controlled and randomized pilot study. An Bras Dermatol. 2012;87(2):203-211. doi: 10.1590/s036505962012000200003.

21. Barrea L, Nappi F, Di Somma C, Savanelli MC, Falco A, Balato A, et al. Environmental Risk Factors in Psoriasis: The Point of View of the Nutritionist. Int J Environ Res Public Health. 2016;13(7):743. doi: 10.3390/ijerph13070743.

22. Johnson JA, Ma C, Kanada KN, Armstrong AW. Diet and nutrition in psoriasis: analysis of the National Health and Nutrition Examination Survey (NHANES) in the United States. J Eur Acad Dermatol Venereol. 2014;28(3):327-32. doi: $10.1111 / j d v .12105$.

23. Naldi L. Risk Factors for Psoriasis. Curr Derm Rep. 2013;2(1):58-65. doi: 10.1007/s13671-012-0034-6.

24. Abrouk M, Lee K, Brodsky M, Nakamura M, Singh R, Zhu TH, et al. Ethnicity affects the presenting severity of psoriasis. J Am Acad Dermatol. 2017;77(1):180-182. doi: 10.1016/j.jaad.2017.02.042.

25. Kim JY, Kang HY, Lee ES, Kim YC. Topical 5-aminolaevulinic acid photodynamic therapy for intractable palmoplantar psoriasis. J Dermatol. 2007;34(1):37-40. doi: 10.1111/j.1346-8138.2007.00213.x.

26. Morton CA, McKenna KE, Rhodes LE. Guidelines for topical photodynamic therapy: update. $\mathrm{Br} J$ Dermatol. 2008;159(6):1245-66. doi: 10.1111/j.13652133.2008.08882.x.

27. Cassell SE, Bieber JD, Rich P, Tutuncu ZN, Lee SJ, Kalunian KC, et al. The modified Nail Psoriasis Severity Index: validation of an instrument to assess psoriatic nail involvement in patients with psoriatic arthritis. $J$ Rheumatol. 2007;34(1):123-9. 